\title{
A Look at the Future
}

\section{Mohamed Bahnassy*}

Soil and Water Sciences Department, AlexandriaUniversity, Egypt

Traditional soil science approach that all we know regarding soil sampling, sample preparation, and soil physical, chemical, and nutritional characterization is well established and recognized by all soil scientists. New approaches regarding mapping soil attributes are relying on the recent advances in soil science, as well as a number of disciplines, among them are computer science, mathematics, statistics, and space sciences. The future might encircle the disappearance of some traditional methods of measurements, as they will be replaced by the spectral characterization of these attributes using hyperspectralhyperspatial remote sensing techniques. This will lead to the availability of ready-to-use high resolution images containing information about soil and vegetation characteristics that will replace traditional laboratory measurements. The approach will be recognized for entirely bare soils or completely covered vegetation canopy. Three issues will rise regarding this technology, the first one will be related to the mix of soilvegetation, and the second one will be linked to the cost of providing such technology, and the third one will be associated with providing knowledge and understanding among researchers about the behavior of different soil and plant constituents in the visible, near infrared, and other parts of the electromagnetic spectrum. By this way, it will easy for scientists to carry out more accurate modeling and predictions about the relationships between soil and the plant that will be revealed in more agricultural production of different crops that will satisfy the need of world population.

One more field of study that will remain on the ground is ethnic soil science, that's related to the indigenous knowledge of the farmers, but the impact of this on other advances will be limited due to its presences in small and scattered areas in the third world countries that still have its own flavor of agricultural practices. On the dark side, this will disappear due to urban encroachment and the displacement of desert people (nomads) or aboriginals to the cities looking for work, money, or developed society.
*Corresponding author: Mohamed Bahnassy, Soil and Water Sciences Department, AlexandriaUniversity, Egypt, Tel. 012-760-9505; Fax: 203-5921260; E-mail: bahnassy@alexu.edu.eg, bahnassy@alex-agr.edu.eg

Received May 24, 2013; Accepted May 28, 2013; Published June 13, 2013

Citation: Bahnassy M (2013) A Look at the Future. J Geophys Remote Sensing 2: e108. doi:10.4172/2169-0049.1000e108

Copyright: (c) 2013 Bahnassy M. This is an open-access article distributed under the terms of the Creative Commons Attribution License, which permits unrestricted use, distribution, and reproduction in any medium, provided the original author and source are credited. 